\title{
Si la libertad, el conocer y el amor son, según Nédoncelle, 'Trascendentales personales'
}

\author{
Juan Fernando Sellés \\ Catedrático de filosofía (Antropología Filosófica) \\ Universidad de Navarra \\ jfselles@unav.es
}

\section{Resumen}

Además de la distinción entre persona y naturaleza, otros temas de vital importancia en la antropología de Nédoncelle son la libertad, el conocimiento, el amor, la intersubjetividad y la relación personal con Dios. En este trabajo nos preguntamos si la libertad, el conocer y el amor personales son, según Nédoncelle, de orden categorial o de orden trascendental, es decir, del orden de las facultades superiores de la persona -la inteligencia y la voluntad-, o si radican en la interioridad personal. Para lograr este objetivo procederemos de la siguiente forma: primero la libertad, pues sin ella no cabe, por una parte, comprender el conocimiento personal de sí y de la intimidad de las demás personas y, por otra parte, tampoco cabe amor personal propio y a los demás. En segundo lugar, el conocimiento propio y ajeno, pues sin él no cabe hablar de amor personal o íntimo. En tercer lugar, el amor, porque es la dimensión superior y, por tanto, para su comprensión se requiere haber reparado en los precedentes.

Palabras clave: Nédoncelle, libertad, conocer, amar, antropología

\section{Abstract}

Besides the distinction between person and nature, other themes of vital importance in Nedoncelle's anthropology are liberty, knowledge, love, intersubjectivity, and the personal relationship to God. In this work we ask if freedom, knowledge and personal love are, according to Nédoncelle, of the categorical or the transcendental order, i.e. of the order of the higher human faculties -intelligence and the will- or if they are rooted in the personal intimacy. To achieve this goal we will proceed as follows: first the liberty, for without it, in one hand, there can be no understanding of personal knowledge of itself and intimacy of others, and, on the other hand, there can neither be personal own love and to others. In second place, own knowledge and otherness, for without it there cannot be any speech of personal or intimate love. In third place, love, because it is the superior dimension, and, therefore, for its comprehension, it is required have noticed the foregoing.

Keywords: Nédoncelle, freedom, knowledge, love, anthropology 


\section{Planteamiento}

En primer término, es conveniente proceder a una aclaración terminológica. Por 'trascendental' se entiende aquí lo que es del ámbito del 'ser' y, por ende, lo que se distingue del 'tener', dicho esto en términos de Marcel; o también, expresado con terminología medieval, lo que pertenece al 'actus essendi' como realmente distinto de la 'essentia'. Si esta distinción real, que caracteriza a toda criatura, se tiene en cuenta en antropología, se debe averiguar qué pertenece a un plano y qué a otro de lo humano. Por tanto, el título de este trabajo pregunta si las dimensiones humanas de la libertad, el conocer y el amor, a las que tanta relevancia dio el pensador parisino, son encuadradas por él en uno y otro plano del hombre.

En un trabajo precedente, en el que tuvimos la oportunidad de mostrar la distinción real entre la persona y la naturaleza humana según Nédoncelle, ${ }^{1}$ la cual responde precisamente a la indicada distinción real entre acto de ser y esencia en el hombre, indicamos que, además de dicho tema, que es el básico para entender los restantes, los otros más relevantes de la antropología de este personalista francés son la libertad, el conocimiento, el amor, la intersubjetividad y la relación personal a Dios. En este trabajo atenderemos al estudio de los tres primeros de esta relación, dejando para una investigación posterior los dos últimos, a saber, el problema de la fundamentación de lo intersubjetivo y la apertura constitutiva del ser humano al divino.

Llevaremos a cabo esta tarea teniendo en cuenta que los libros más representativos de la antropología del filósofo galo para estos temas son, por orden cronológico: a) La reciprocidad de las consciencias. Ensayo sobre la naturaleza de la persona (1942); b) Hacia una filosofía del amor y de la persona (2ª ed., 1957); c) Conciencia y logos. Horizontes y métodos de una filosofía personalista (1961); d) Persona humana y naturaleza. Estudio lógico y metafísico (2aㅡ. ed., 1963); e) Exploraciones personalistas (1970), y f) Intersubjetividad y ontología. El desafío personalista (1974).

En la explicación de los temas indicados procederemos según el siguiente orden expositivo: primero la libertad, pues sin ella no cabe, por una parte, comprender el conocimiento personal de sí y de la intimidad de las demás personas y, por otra parte, tampoco cabe amor personal propio y a los demás. En segundo lugar, el conocimiento propio y ajeno, pues sin él no cabe hablar de amor personal o íntimo. En tercer lugar, el amor, porque es la dimensión superior y, por tanto, para su comprensión se requiere haber reparado en los precedentes. La pregunta de la que partimos en este estudio, y que trataremos de responder, es si en la antropología de Nédoncelle la libertad, el conocer y el amor humanos son de orden 'trascendental' o de orden

${ }^{1}$ Cf. Sellés, J. F., “La distinción entre persona y naturaleza según Nédoncelle”, en Metafísica y persona, núm. 9, 2013. 
'categorial', es decir, si -teniendo en cuenta su distinción real entre persona y naturaleza en el hombre- pertenecen a la primera o a la segunda dimensión humana.

\section{Si la libertad es 'trascendental'}

a) En La reciprocidad de las conciencias Nédoncelle escribe que:

G. Marcel observa justamente que tenemos una libertad de rechazo o de adhesión proporcional a la densidad metafísica de la realidad que se presenta a nuestro espíritu: así, es más fácil negar a Dios que la materia. Se puede añadir que, por la misma razón, el espíritu está expuesto a volverse contra él mismo más brutalmente que como lo haría contra las cosas. Este extravío es también más grave... El mal moral más profundo no es el desgarramiento asumido por la conciencia, sino la perversidad voluntaria. Podemos distinguir las siguientes direcciones de la voluntad rebelde: la indiferencia, la voluntad de poder y el odio. ${ }^{2}$

En el texto que precede se advierte que el autor distingue entre dos intensidades de la libertad, una inferior, que se ejerce respecto de las realidades materiales, y otra superior, que se emplea con las espirituales. Pero no dice explícitamente en qué nivel de lo humano engarza cada una de ellas. Con todo, es claro que Nédoncelle las vincula a la voluntad, y no menos claro que ésta es una 'potencia' de la persona, no la 'persona', pues es manifiesto que nadie se reduce a su voluntad y, precisamente por eso, cada quien puede orientar a esta potencia suya en una u otra dirección. En consecuencia, si se vincula la libertad a la voluntad, no parece que radique en la intimidad de la persona, es decir, que sea de orden 'trascendental' o del ser, sino 'categorial' o del tener. Sin embargo, Nédoncelle añade en esta publicación que "la libertad es una respuesta de la que depende nuestro destino", ${ }^{3}$ lo cual parece indicar que, al menos por su relevancia, no se trata de una dimensión humana periférica,

\footnotetext{
${ }^{2}$ Cf. Nédoncelle, M., La reciprocidad de las conciencias, Madrid: Caparrós, 1996, p. 189. El autor explica así las tres direcciones de la voluntad rebelde:

1) “La indiferencia es una primera desviación del orden personal. Responde a un ideal de descenso hacia la naturaleza", p. 189. "El primer abandono de la generosidad es la indiferencia. Por ella, la mayoría de los pensadores griegos querían obtener la felicidad. Esta actitud exige una renuncia al personalismo", p. 189.

2) "La voluntad de poder no puede realizarse completamente ni de derecho ni de hecho, pues no puede eliminar el tú como causa del yo". p. 190. "La voluntad de poder es egoísta; o para utilizar un nombre más exacto, es orgullosa: su finalidad y su alegría son vencer las resistencias, someter y dominar las conciencias", p. 190. "El yo nunca se separa totalmente de Dios y, en este aspecto, la voluntad de poder sufre un peor fracaso metafísico que la indiferencia. Ésta es posible en teoría, aquella no lo es... Pero esta voluntad se puede realizar a medias; y crea entonces un contra-universo de cualidades hostiles a la persona", p. 191.

3) “Opuesto a la indiferencia y a la voluntad de poder, el odio apunta el tú al corazón como al amor", p. 192. “Intenta ir hasta el centro de la conciencia. Se puede detestar la máscara, no odia más que a la personalidad", p. 192. "Hay muchas clases de odio: con motivo o gratuito, simple, insultante, sádico, sagrado, unilateral y bilateral, etc.", p. 193. "El odio perfecto quiere el mantenimiento y la promoción infinitas del tú para poder continuar rebelándose y odiar. Quiere, como el amor, la eternidad del otro", p. 195. "El odio no puede escapar, sin embargo, a un punto de ignorancia central y a una ausencia de comunión por parte de quien odia", p. 196.
}

\footnotetext{
${ }^{3}$ Nédoncelle, M., La reciprocidad de las conciencias, p. 247.
} 
sino nuclear. En esta misma dirección cabe leer otra afirmación suya: "el mayor misterio de la creación es la libertad derivada", ${ }^{4}$ pues si lo es, debe tratarse de una realidad central. Al final agrega que "la libertad es plural: se anuda y arraiga en la perfección a causa de la pluralidad mutua que es necesaria y proporcionada a su existencia", 5 de modo que, dada tal pluralidad, se puede sospechar que está vinculada a las diversas dimensiones humanas, aunque el autor lo deje sin explicitar.

b) Por otra parte, en su libro Vers une philosophie de l'amour et de la personne se lee que "mi libertad es mi persona", ${ }^{6}$ y es sabido que la 'persona' equivale, para Nédoncelle, a la interioridad o el espíritu, es decir, a la novedad e irrepetibilidad de cada quien. Por tanto, parece considerar que la libertad es del ámbito trascendental. En esta misma obra, en el apartado "¿Se comunica la libertad?", escribe que "ser libre es elevarse a sus causas, tomar en la mano el propio destino, llegar a ser creador de sí y por sí", y redacta, asimismo, que la libertad es "la culminación de una persona concreta", ${ }^{8}$ lo cual parece ir en la misma línea que lo precedente. Indica también que "ser libre y ser suyo es todo uno", 9 de manera que parece tomar 'persona' y 'libertad' como equivalentes.

c) En Conscience et logos. Horizons et méthodes d'une philosophie personnaliste, no encontramos pasajes de relieve referentes a la libertad.

d) Por otro lado, en Personne humaine et nature. Étude logique et métaphysique, en el apartado "La libre causalidad del yo encarnado", Nédoncelle indica que "hay una libertad primitiva de mí en Dios", ${ }^{10}$ es decir, que la libertad existe en nosotros como un don divino original. Añade que la libertad es "la necesidad de determinar el don primitivo, la libertad inicial es una espontaneidad que se complace en sí misma, implicada profundamente en el acto que nos hace ser". ${ }^{11}$ De modo que, ante estos textos, no parece que estemos ante una comprensión 'categorial' de la libertad, sino ante otra de cuño 'trascendental'. Además de esa aludida 'necesidad de determinar el don inicial', indica que hay dos dimensiones en la libertad: una nativa y otra

\footnotetext{
${ }^{4}$ Nédoncelle, M., La reciprocidad de las conciencias, p. 303.

${ }^{5}$ Nédoncelle, M., La reciprocidad de las conciencias, p. 305.

6 “Ma liberté est ma personne". NéDoncelle, M., Vers une philosophie de l'amour et de la personne, Paris: Aubier, 2ª ed., 1957, p. 124.

7 "Être libre, c'est s'arracher à ses causes, prendre en main son propre destin, devenir créateur de soi par soi". NÉDONCELle, M., Vers une philosophie..., p. 135.

${ }^{8}$ [La liberté est] “l'accomplissement d'une personne concrète”. NÉDoncelle, M., Vers une philosophie..., p. 136.

9 “Être libre et être soi, c'est tout un”. NéDoncelle, M., Vers une philosophie..., p. 136.

${ }^{10}$ Nédoncelle, M., Personne humaine et nature. Étude logique et métaphysique, Paris, Aubier, $2^{\text {a }}$ ed., 1963, p. 145.

${ }^{11}$ [La liberté] "est la nécessité de déterminer la donnée primitive, la liberté initiale est une spontanéité complaisante à elle-même; impliquée si profondément dans l'acte qui nous fait être". NÉDonCELLE, M., Personne humaine et nature..., p. 145.
} 
destinal. Ahora bien, tanto una como otra son de ámbito trascendental. Describe la primera, la primitiva o nativa, como un querer espontáneo que no puede ni declinar ni disminuir, que es el inicio de todos nuestros actos y que es necesaria. ${ }^{12}$ Explica la segunda, la derivada, como aquélla que decide el destino personal humano. ${ }^{13}$ Expone que entre una y otra existe una discontinuidad, ${ }^{14}$ intervalo que entiende como fisura o distancia entre lo imperfecto y lo perfecto. ${ }^{15}$ Agrega que de la segunda, que no está determinada, ${ }^{16}$ depende el cumplimiento del 'yo ideal' o vocación que Dios quiere para cada uno. ${ }^{17}$ Que ambas son distintas es manifiesto porque la segunda se puede oponer a la primera, ${ }^{18}$ aunque no la pueda aniquilar. ${ }^{19}$ Por lo demás, la libertad humana no se reduce, según Nédoncelle, al 'libre albedrío', tal como éste fue entendido en la filosofía medieval y por los pensadores del s. xx que siguieron tal estela. ${ }^{20}$ Por lo mismo, tampoco considera que el ejercicio del libre albedrío sea el fin de la libertad. ${ }^{21}$ Ahora bien, como es claro que el libre albedrío (el dominio sobre nuestros actos) es de orden categorial, se puede sospechar que la libertad irreductible a él, a la que apunta este personalista, es de orden 'trascendental'.

e) Por otro lado, en su publicación Explorations personnalistes Nédoncelle marca la afinidad que el diálogo mantiene con la libertad ${ }^{22}$ y el que la dialéctica guarda con

\footnotetext{
12 “La liberté primitive est un vouloir spontané. 148. “La liberté primitive ne pouvait déchoir ni diminuer... Elle n'est que le début éternel de tous nos actes; et le moi, en vertu même de cet élan intérieur, doit connaître la liberté contingente d'après la liberté nécessaire". NÉDoncelle, M., Personne humaine et nature..., p. 148.
}

13 “L'intériorité même que Dieu lui donne l'oblige en fait à mettre un intervalle entre Dieu et elle-même... C'est de cette façon qu'elle doit décider de sa destinée". Nédoncelle, M., Personne humaine et nature..., p. 148.

14 "Entre la liberté primitive et la liberté dérivée, ou réponse que la personnalité se donne à elle-même, il y a discontinuité ou contingence". NÉDONCELLE, M., Personne humaine et nature..., p. 149.

15 “Il y a dans notre être une fissure, une cassure même; et c'est pourquoi toute psychologie du moi reste fatalement dans l'inachèvement et la dualité". Nédoncelle, M., Personne humaine et nature..., p. 149.

16 "La liberté dérivée prolonge certes à plus d'un élan primitif. Mais elle ne se guide pas sur un fatum divin". NÉDONCELLE, M., Personne humaine et nature..., p. 149.

17 “Réagissant sur le mou idéal, la réponse libre réagit sur le toi éternel, c'est-à-dire sur un aspect de Dieu même, et modifie à chaque instant la nuance de l'identité dans le nous conséquent... Le vouloir divin et l'image primitive sont immutables: la modification causée par l'homme, surtout s'il s'agit d'une réponse en désaccord avec le moi idéal, ne peut qu'être unilatérale". NéDoncelle, M., Personne humaine et nature..., p. 150.

18 “La liberté dérivée est en quelque sorte une inversion de la liberté radicale. C'est une liberté qui se peut tourner contre le liberté". Nédoncelle, M., Personne humaine et nature..., p. 151.

19 “Le liberté infidèle reste suspendue à la liberté qui veut la liberté de l'essence primitive... Il subsiste en elle un minimun de garantie indestructible qui est l'image primitive du moi". NéDonCelle, M., Personne humaine et nature..., p. 151. 20 "Pour une personnalité incarnée, la liberté commence dans un libre arbitre, mais elle ne s'y réduit sans doute jamais". Nédoncelle, M., Personne humaine et nature..., p. 151.

21 "La choix est la condition de la liberté humaine, mais non pas son but, qui est l'invention et l'achèvement de notre innéité". 152.

22 “Par là se découvre l’affinité du dialogue pour la liberté, de même que nous avions observé l'affinité de la dialectique pour la nécessité". NÉDoncelle, M., Explorations personnalistes, Paris: Aubier, 1970, p. 81. 
la necesidad, a la par que señala la convergencia existente entre el amor y la libertad. ${ }^{23}$ Pero no se pronuncia acerca de si tal libertad es íntima, además de manifestativa.

f) Por último, en Intersubjectivité et ontologie. Le défi personnaliste, ya en el Prólogo, afirma que la persona es la garante de la libertad, pues se desaferra de los sistemas imperantes. ${ }^{24}$ En ese mismo inicio añade que "si el personalismo es verdadero, debe tener un fundamento, pero éste debe ser la expresión de una libertad". ${ }^{25}$ Más adelante, en un artículo de 1974: "Le sacré et la profanation", de los muchos que componen este libro, escribe que "lo sagrado es la presencia de lo último en el seno de la libertad si hay un Creador de las libertades y una comunidad posible de personas". ${ }^{26}$ En una de las 'conclusiones' de otro artículo, en este caso de 1973, “De la foi comme connaissance dans l'histoire de la philosophie occidentale: genèse d'un sen intersubjectif", alude a la libertad del yo y del tú. ${ }^{27}$ Más adelante, en el artículo "Les variations de Boèce sur la personne", de 1955, indica que, entre las seis descripciones boecianas de 'persona', hay una según la cual se la describe como "una vocación a la vida divina por la razón, el amor y la libertad". ${ }^{28}$ También en el artículo "La notion de personne dans l'œuvre de Saint Anselme", de 1959, señala que San Anselmo sigue tres líneas para describir a la persona, siendo según Nédoncelle la más interesante, porque indica perfección, su doctrina propia, la cual está fundada sobre la libertad y dignidad espiritual. ${ }^{29}$ Cabe destacar, por último, que en la "Remarque finale: les deux formes de la relation", una especie de apéndice a este libro, se lee que "la persona no es una cosa sino una vocación y una libertad", ${ }^{30}$ sentencia que, de forma más explícita que las precedentes, impele a incluir la libertad en el orden trascendental.

\footnotetext{
23 "Il y a convergence entre l'amour, la liberté et l'éternité des personnes". NéDonCELLE, M., Explorations personnalistes, p. 88.

24 “La personne est anti-idéologique par essence: elle est notre seule garantie de liberté et de dégagement, face aux systèmes qui l'étoufferaient". NÉDONCELLE, M., Intersubjectivité et ontologie. Le défi personnaliste, Louvain: Nauwelaerts, 1974, p. 1.

${ }^{25}$ NÉDONCELLE, M., Intersubjectivité et ontologie..., p. 4.

${ }^{26}$ Nédoncelle, M., Intersubjectivité et ontologie..., p. 168.

${ }^{27}$ Cf. NÉDoncelle, M., Intersubjectivité et ontologie..., p. 234.

${ }^{28}$ NÉDoncelle, M., Intersubjectivité et ontologie..., p. 267.

${ }^{29}$ Cf. Nédoncelle, M., Intersubjectivité et ontologie..., p. 286.

30 “La personne n'est pas une chose mais une vocation et une liberté". NéDONCELLE, M., Intersubjectivité et ontologie..., p. 276.
} 


\section{2. ¿Es el conocimiento personal propio y ajeno 'trascendental'?}

El tema del conocimiento de la propia intimidad y de la ajena es en Nédoncelle más complejo que el de la libertad, y también más extensamente tratado por él. A tal conocer le llama muchas veces 'conciencia'.

a) En La reciprocidad de las conciencias, expone que "ciertamente la conciencia, y en particular la conciencia de sí, están unidos a la persona. Pero todo hace pensar que la percepción del otro es solidaria de la percepción de sí". ${ }^{31} \mathrm{Si}$ tal conocer está unido a la persona, cabe suponer que no cabe persona sin tal conocer. Con todo, si se pregunta de qué tipo de conocimiento se trata, el pensador francés responde que es claro que no es ningún conocer racional, sea éste del tipo que sea. Así, no es un conocer por analogía. ${ }^{32} \mathrm{Y}$, por supuesto, aunque el conocimiento propio comience por los sentidos, ${ }^{33}$ es irreductible al conocer sensible. ${ }^{34}$ Se trata, más bien, de un conocimiento intuitivo, ${ }^{35}$ en modo alguno objetivo, ${ }^{36}$ es decir, según 'objeto pensado' o idea. Nédoncelle estima que la intimidad personal no es compuesta y que, asimismo, tampoco lo es el conocimiento que la alcanza. ${ }^{37}$ En alguna ocasión indica que tal conocer

\footnotetext{
${ }^{31}$ Nédoncelle, M., La reciprocidad de las conciencias, p. 14.

32 “El razonamiento por analogía no nos hace conocer ni la existencia ni la naturaleza personal del otro". NÉDONCELLE, M., La reciprocidad de las conciencias, p. 37. “La analogía, por hipótesis, supone parecidos y diferencias entre los términos que construye y compara. Ella se queda dudosa e indecisa. Sin duda recurrimos a ella en la percepción banal, que es indiferente a la singularidad personal. Pero lo que nuestras interpretaciones inductivas rastrean y así buscan determinar es la máscara del otro más que el otro mismo". NéDoncelle, M., La reciprocidad de las conciencias, pp. 38-39. 33 “La presencia de nuestro yo comienza con el condicionamiento sensible. No hay percepción espiritual que se forme sin percepción natural o su ocasión. No podemos abandonar un aspecto de nuestra esencia que pese a su humildad es la provisión de ruta del espíritu". NéDoncelle, M., La reciprocidad de las conciencias, p. 160. Más adelante añade: "Il est encore une raison pour laquelle nous rejetterons l'étiquette idéaliste: c'est que la personne nous arrive toujours à travers la nature. L'esprit finit par toucher l'esprit, mais il commence par le chemin des corps et de l'âme animale". NéDoncelle, M., La reciprocidad de las conciencias, p. 119.

34 "El conocimiento personal se obtiene por mediaciones naturales, pero sin reducirse nunca a éstas". NÉDONCELLE, M., La reciprocidad de las conciencias, p. 71. "La aparición de nuestra conciencia personal es imposible explicarla por la naturaleza, pero su desarrollo todavía lo es más". NéDoncelle, M., La reciprocidad de las conciencias, p. 119.

35 “Toda percepción del otro es una promoción intuitiva de su ser". Nédoncelle, M., La reciprocidad de las conciencias, p. 39.

36 “Decir que la relación de conocimiento es una relación de contrariedad (como los presocráticos), o de similitud (como Platón y la mayor parte de los teóricos después de él), es situarse en la categoría del objeto y no en la comunicación transubjetiva; es hablar de la naturaleza y no del tú". NéDoncelle, M., La reciprocidad de las conciencias, p. 42. 37 "Una persona no se divide; se deja penetrar más o menos, pero en conjunto, debe ser conocida como una originalidad total bajo pena de no ser conocida en nada". NÉDoncelle, M., La reciprocidad de las conciencias, p. 62. "Si el conocimiento de la persona es indivisible, la realidad de la persona lo será con mayor razón. No tiene partes propiamente dichas, y no puede tampoco 'formar parte' de otro centro personal". NéDonCelLe, M., La reciprocidad de las conciencias, p. 63.
} 
es un hábito. ${ }^{38}$ Como es sabido, 'hábito' deriva del latín 'habere', que significa 'tener'; de acuerdo con esto, se trataría, pues, de un conocer posesivo. Pero a veces sospecha que es algo más que un 'tener': "A la inversa que los realistas, se puede estimar que el ser y el conocer coinciden en cierto sentido. Para cada nivel del ser corresponde un nivel de conocer", es decir, que tal conocer está, en cierto modo, en el plano del 'ser', 39 aunque no coincida enteramente con el ser. ${ }^{40}$ Nédoncelle sostiene que el conocimiento propio siempre es inferior al propio ser y lo es, asimismo, el lenguaje que expresa tal conocer: "lo que digo de mí no soy exactamente yo, pues yo soy más acá o más allá de lo que digo cuando lo expreso; el lenguaje es un retraso y el pensamiento una corriente que pasa". ${ }^{41}$ A veces a tal conocer lo designa como sabiduría:

la sabiduría de los sabios es a menudo la última conquista de la existencia temporal. Y nos es dada para guiarnos en la noche. No le corresponde, pues, a ella cantar la luz de la mañana y la juventud del mundo que no pasa. Pero le basta con estar próxima al objetivo y no haber sido indigna del espíritu del cual da testimonio. ${ }^{42}$

b) Por otra parte, en Vers une philosophie de l'amour et de la personne, Nédoncelle achaca a Kant el no haber facilitado el conocimiento de la persona y de la intersubjetividad, ${ }^{43}$ porque lo conocido, según el pensador de Königsberg, son representaciones, pero para el filósofo de Roubaix la persona no se conoce formando representaciones de ella, ya que la persona, más que una representación, es una 'presentación',44 que se hace accesible a sí misma como fruto de una entrega del Creador, ${ }^{45}$ y se deja conocer asimismo por las demás personas si se entrega a ellas. ${ }^{46}$ Por eso el solipsismo es absurdo, porque impide conocer el sentido personal propio y ajeno. ${ }^{47}$ De manera que sin Dios no se puede alcanzar a descubrir el completo

\footnotetext{
38 "Esta memoria elemental que retiene al yo en él mismo en el transcurso de su expansión puede ser llamada hábito". NéDONCELle, M., La reciprocidad de las conciencias, p. 67.

${ }^{39}$ Nédoncelle, M., La reciprocidad de las conciencias, p. 146.

40 “Notre conscience n'est pas tout note être". Nédoncelle, M., La reciprocidad de las conciencias, p. 192.

${ }^{41}$ Nédoncelle, M., La reciprocidad de las conciencias, p. 183.

${ }^{42}$ NéDoncelle, M., La reciprocidad de las conciencias, p. 306-7. Las alusiones a la 'noche' y la 'mañana' están en deuda con las respectivas metáforas nietzscheanas.
}

${ }^{43}$ Cf. Nédoncelle, M., Vers une philosophie de l'amour et de la personne, p. 101.

44 “La donnée (dato) ... est une présentation et non une représentation”. Nédoncelle, M., Vers une philosophie de l'amour et de la personne, p. 102.

45 “En prenant conscience de moi-même, je prends en réalité conscience que mon cogito même m’est donné: je pense, donc je suis pensé; je suis, c'est-à-dire je suis voulu. Mais pour désigner le donateur dans le don de mon acte constitutif, pour m'assurer qu'il est un Créateur vivant plutôt qu'une valeur anonyme, il faut une dialectique qui nous conduirait bien au-delà du fait psychologique initial dont nous étions partis et qui était la réciprocité humaine". NÉDONCELle, M., Vers une philosophie de l'amour et de la personne, p. 110.

46 “La personne n'est pas accessible si elle ne se donne pas d'une certaine manière à qui la contemple”. NÉDONCELLE, M., Vers une philosophie de l'amour et de la personne, p. 109.

47 "Si le solipsisme est absurde, c'est que la saisie concrète de mon acte est du même coup l'intuition des influences humaines qui m'ont promu depuis le berceau... Je ne peux nier autrui sans me nier... L'acte par lequel je suis est 
sentido personal, y esto, que ha sido olvidado en teoría del conocimiento -como remarca este pensador parisino-, no es una afirmación prestada de la fe sobrenatural, sino fruto del saber natural. ${ }^{48}$ Debido a este olvido, este filósofo francés no está de acuerdo ni con la gnoseología del realismo ingenuo, ni tampoco con la del idealismo crítico. Por ello se enfrenta también con el objetivismo de Husserl.

c) Por lo que respecta a su obra Conscience et logos. Horizons et méthodes d'une philosophie personnaliste, en ella Nédoncelle afirma que el método adecuado para el propio conocimiento es la 'reflexión'. ${ }^{49}$ Ésta no es de orden racional sino superior, intelectual, ${ }^{50}$ aunque también las ideas formadas por la razón manifiesten de alguna manera el ser personal. ${ }^{51} \mathrm{El}$ conocimiento intelectual es intuitivo, directo, sin mediaciones, y va al núcleo de lo real, no a los aledaños. ${ }^{52}$ Reitera, como en la obra precedente, que, al ser la persona 'relación', su conocimiento tiene que ser relacional, no solitario: "la conciencia personal no puede alcanzarse en un cogito perfectamente solitario; encuentra en ella y con ella una sociedad, un mundo, un Dios. Pero eso que ella encuentra, que comprende, está frecuentemente a distancia de su intimidad". 53

l'acte même par lequel on me fait être. L'autonomie que je me donne est ontologiquement à la mesure des présences que j'ai reçues". Nédoncelle, M., Vers une philosophie de l'amour et de la personne, p. 111.

48 "Notre dessein est de montrer qu'au point de départ un donné était présent qui réunissait les sujets dans une vocation de donateurs. Ce fait primitif n'est pas posé par la foi et l'espérance, mais par l'expérience et la raison. Il a été singulièrement méconnu au grand détriment de la théorie de la connaissance et de la métaphysique de la personne". Nédoncelle, M., Vers une philosophie de l'amour et de la personne, p. 113.

49 "La vérité subjective est une sincérité du savoir; elle est objective quand elle a un contenu; et son premier contenu est celui qu'elle tire d'une réflexion sur elle-même". Nédoncelle, M., Conscience et logos. Horizons et méthodes d'une philosophie personnaliste, Aubier: París, p. 35. Nédoncelle señala varios equívocos para entender la 'reflexión' e indica que la buena comprensión de ella oscila entre los dos extremos siguientes: 1) ¿Está entre el recogimiento y la dispersión? Está más cerca del primero. 2) ¿Es percepción o análisis? Tiene dos maestros: la percepción y el análisis. 3) ¿Es conocer impersonal o personal? Tiene que ver con el conocer impersonal y con el personal, porque el primero es una máscara del segundo. 4) ¿Es detención o una vía más lúcida del espíritu? Ambas. 5) ¿Es reflexión solitaria o interpersonal? Las dos.

50 “Tout ce qui est personnel a quelque chose d'intellectuel”. Nédoncelle, M., Conscience et logos..., p. 40.

51 “Toutes les idées, en dépit des apparences, peuvent traduire en leur structure l'être personnel”. NéDonCELLE, M., Conscience et logos..., p. 37. "L'impersonnalité des idées, même dans la science, n'est que relative". NÉDonCELle, M., Conscience et logos..., p. 37.

52 "L'intuition est la connaissance de une chose, le raisonnement est la connaissance des alentours d'une chose". Nédoncelle, M., Conscience et logos..., p. 119.

${ }^{53}$ Nédoncelle, M., Conscience et logos..., p. 63. Por lo demás, el amplio capítulo de este libro dedicado al 'principio de correspondencia y el principio de correctibilidad entre filosofías' lo conforman diversas consideraciones sobre teoría del conocimiento que no versan directamente sobre nuestro tema. Por su lado, en el capítulo "Logos y persona" indica que hay tres sentidos de logos: el judío, como palabra, el griego, como pensamiento, y el cristiano, como Cristo. Añade que en latín logos tiene dos sentidos: verbum y ratio, y que de ahí derivan dos líneas especulativas: la que va a las personas trinitarias y la que va a las ideas. Para nuestro tema, lo que interesa de este apartado es que "existe una íntima conexión entre el Logos y la persona" (NÉDonCelle, M., Conscience et logos..., p. 231), porque logos indica relación, y -ya se ha indicado- que también la persona tiene ese significado. 
d) En Personne humaine et nature. Étude logique et métaphysique, Nédoncelle sostiene que "el yo humano no puede conocerse reflexivamente más que llevando su pensamiento y su ser a un principio supremo de pensamiento y de ser que sea él mismo personal", ${ }^{54}$ es decir, a Dios. En efecto, el completo sentido personal sólo se alcanza si el ser divino nos lo manifiesta. Niega que el conocimiento del sujeto se lleve a cabo mediante 'ideas generales'. ${ }^{55}$ Además, "los aspectos conceptuales de la persona implican nociones negativas", ${ }^{\prime 6}$ porque el lenguaje está sacado de la naturaleza y la persona no es naturaleza. Por tanto, el concepto no es apto para el conocimiento personal. Por su parte, si se intenta conocer a la persona mediante el juicio racional, este pensador advierte que en el juicio el predicado no expone todo lo que es el sujeto. Afirma asimismo que "todo juicio se separa de la simplicidad intuitiva por la admisión de una generalidad", ${ }^{57}$ por tanto, tampoco este acto racional es apto para conocer a la persona. En cambio, "en la intuición personal, el sujeto y el atributo no tienen ningún carácter conceptual, y la vida y el pensamiento se reconcilian silenciosamente" ${ }^{58}$ Por el contrario, los juicios psicológicos objetivan un yo y, en consecuencia, tampoco son aptos para el conocimiento personal. ${ }^{59}$ Todos ellos son posesivos, pero "el tener del sujeto es el envoltorio del acto". ${ }^{60}$ Tras sostener que el principio de identidad, el de contradicción y el de 'tercio excluso' no sirven para aplicarlos en el caso de la persona, Nédoncelle concluye que el conocer intuitivo es distinto del racional, y es el que sirve para conocer a las personas. ${ }^{61}$ El conocer racional no sirve porque es posesivo: "la objetividad está unida al tener... el conocimiento objetivo implica una forma especial de relación: la del tener. Por ella poseo o limito el objeto". ${ }^{62}$ Pero lo poseído por tal conocer es ideal, no real. De modo que si se pretende conocer así al sujeto, se conoce la idea de sujeto, pero la idea de sujeto no es el sujeto. La idea deja al sujeto supuesto y no ahonda en él: “toda afirmación de la objetividad supone la ficción intelectual de una sustancia al menos ésa del sujeto que tiene el objeto". ${ }^{63}$ Nédoncelle advierte la limitación no ética que supone el conocimiento objetivo. Formar ideas de la persona no es progresar en el conocer sobre la

\footnotetext{
${ }^{54}$ Nédoncelle, M., Personne humaine et nature. Étude logique et métaphysique, p. 13.

55 “La connaissance du sujet par lui-même ne comporte pas d'idées générales”. NéDonCELle, M., Personne humaine et nature..., p. 45.

56 Nédoncelle, M., Personne humaine et nature..., p. 47.

57 Nédoncelle, M., Personne humaine et nature..., p. 53.

58 Nédoncelle, M., Personne humaine et nature..., p. 54.

59 “Le jugement psychologique ne peut cependant s'affranchir des obstacles généraux du jugement". NÉDONCELLE, M., Personne humaine et nature..., p. 59.

${ }^{60}$ Nédoncelle, M., Personne humaine et nature..., p. 66.

${ }^{61}$ Cf. Nédoncelle, M., Personne humaine et nature..., p. 99.

62 Nédoncelle, M., Personne humaine et nature..., p. 110-111.

63 Nédoncelle, M., Personne humaine et nature..., p. 112.
} 
persona, sino un descenso del espíritu. ${ }^{64}$ Ahora bien, "no todo conocimiento es objetivo. La intuición de las conciencias que comunican comporta una distinción, pero no una objetividad". ${ }^{65}$ Por vía de objetividad lo que se consigue es, a lo sumo, tener un conocimiento simbólico de la persona. ${ }^{66}$ En suma, la objetividad (objetos mentales) no alcanza a conocer la persona, porque sirve para conocer lo que está más allá del objeto pensado, no lo que está más acá de él:

[...] la objetividad es un análisis o una síntesis, pero no es necesariamente penetrada de intuición o puesta al servicio de la intuición; es normalmente un esquema destinado a la acción sobre la naturaleza. Se aplica a las personas en la medida en que están encarnadas en la naturaleza y sometidas a las leyes de la acción exterior, ${ }^{67}$ pero no penetra en su interioridad.

e) Por otra parte, en Explorations personnalistes, ante la objeción de que yo no me puedo conocer como persona y de que no puedo conocer a las demás personas más que por la percepción sensible, Nédoncelle afirma: "admitimos sin reserva que la persona humana está encarnada y que para conocerla pasamos por un no-yo de orden sensible. Pero sostenemos que este rodeo no modifica en sustancia la originalidad de la persona y de su intuición. Incluso si, por ejemplo, el cogito nace en un conocimiento sensible, se sustrae también a la alienación de lo sensible". ${ }^{68}$ Como se puede apreciar, Nédoncelle afirma que lo sensible en nosotros no es la persona que somos, sino un modo de estar de la persona.

f) Por último, en el Prólogo de Intersubjectivité et ontologie. Le défi personnaliste, Nédoncelle afirma que "el cogito revela una consustancialidad del espíritu y de la persona". ${ }^{69}$ Pero si se pregunta si acaso es tal cogito el que es susceptible de conocer el espíritu y la persona, su respuesta es que no enteramente, pues "la persona escapa a eso que ella reconoce o a eso que ella descubre". ${ }^{70}$ Más adelante, en este mismo trabajo, explica que nuestra conciencia es finita, lo cual significa que el acto de conocer sólo posee una forma conocida. ${ }^{11}$ Pero añade que "quien dice finitud dice encuentro de un límite... quien dice límite dice presencia de un elemento negativo". ${ }^{72}$ En otras

64 “Dans l'objectivité de la connaissance, l'instrumentalité est d'abord un retrait; l'avoir n'est pas une libération mais une descente de l'esprit dans les qualités. L'esprit ne pouvant se borner à cette passivité qui abaisse son acte, trace une frontière et sépare de lui la qualité. Il crée l'extrinsèque; il se dégage de la passivité par l'image et le concept, et il libère les choses pour essayer de les dominer". Nédoncelle, M., Personne humaine et nature..., p. 112-3.

${ }^{65}$ NÉDoncelle, M., Personne humaine et nature..., p. 115.

66 “L'avoir comme objectivité n'est apte qu'à représenter symboliquement la présence personnelle, puisque l'ordre des personnes y est ramené à celui des choses". Nédoncelle, M., Personne humaine et nature..., p. 115.

${ }^{67}$ NÉDoncelle, M., Personne humaine et nature..., p. 116.

${ }^{68}$ NéDonCelle, M., Explorations personnalistes, p. 55.

${ }^{69}$ NéDONCELLE, M., Intersubjectivité et ontologie..., p. 4.

70 “La personne échappe à ce qu'elle subit ou à ce qu'elle découvre”. NÉDONCELLE, M., Intersubjectivité et ontologie..., p. 5.

${ }^{71}$ Cf. Nédoncelle, M., Intersubjectivité et ontologie..., p. 99.

72 NÉdonCelLe, M., Intersubjectivité et ontologie..., p. 99. 
palabras: notar que el conocimiento objetivo es limitado supone saltar el límite, lo cual impele a decir que queda mucho por conocer de nosotros mismos. Tras ello el reto noético radica, por tanto, en progresar en el propio desvelamiento. Afirma que las filosofías que no albergan este leitmotiv entran en crisis, y "la crisis comienza por una cierta disociación entre la conciencia y la persona". ${ }^{73}$ En un artículo de los que se compone esta obra, "Le sacré et la profanation", de 1974, propone -more Schelerotro tipo de conocimiento personal: el sentimiento, pues escribe que "el sentimiento de lo sagrado surge cuando el misterio de nuestra persona o de otro existente está a punto de desvelarse a nuestra conciencia... El sentimiento de lo sagrado presenta este carácter paradójico de ser funcionalmente cognitivo escapando radicalmente al concepto". ${ }^{74}$ En otro artículo, "De la foi comme connaissance dans l'histoire de la philosophie occidentale: genèse d'un sen intersubjectif", de 1972, propone -more Jacobi y Coleridge- otro modo de conocer la persona: la fe, pues -frente a Kierkegaard- estima que ésta es una forma de conocimiento que da acceso a la esfera intersubjetiva de la existencia, ${ }^{75}$ mientras que mantiene que el conocimiento propio se debe, más bien, a la intuición. En suma, sentimiento, fe, intuición, son diversos métodos de acceso al conocimiento personal que Nédoncelle ensayó. ${ }^{76}$ Es de destacar asimismo que en el artículo "Le rôle de l'oubli de soi dans la formation de la personne d'après Rosmini", de 1957, Nédoncelle reitera que "nuestra conciencia de nosotros mismos es de ordinario desbordada por el objeto primordial que nos constituye en nuestra subjetividad personal", 77 a lo que añade que "no tenemos otro modo de llegar al conocimiento del alma que a partir del yo", ${ }^{\prime 8}$ afirmación que supone al yo como distinto del alma, pero como lo más cercano a ella y como su acceso noético. Por último en "L'intersubjectivité d'après Buber et Lévinas", artículo de 1974, Nédoncelle se opone a estos dos representantes de la filosofía del diálogo por su concepción de la racionalidad, ya que ellos consideraban que todo pensamiento es posesivo, mientras que él piensa que también es oblativo.

\footnotetext{
73 Nédoncelle, M., Intersubjectivité et ontologie..., p. 121.

74 NÉDONCELLE, M., Intersubjectivité et ontologie..., p. 157.

75 Cf. Nédoncelle, M., Intersubjectivité et ontologie..., p. 232.

${ }^{76}$ En las Conclusiones del citado artículo Nédoncelle resume, entre otras cosas, que: “1) la foi entendue comme connaissance de la personne est rarement parvenue à une signification nette et constante, mais elle a assez souvent été présente dans la langue philosophique sous forme occasionnelle et implicite. 2) Quand elle est appliquée à la connaissance de la personne, la foi l'est surtout à la connaissance d'autrui; la saisie du moi lui-même est d'ordinaire considérée comme une intuition et désignée par un autre terme que la foi ou la croyance. 3) Entre la foi cognitive et la raison, ce qui fait souvent l'intermédiaire depuis Descartes, Malebranche et Maine de Biran, c'est le 'sentiment intérieur', terme autour duquel on a groupé de préférence ce qui concerne la conscience de soi". NÉDoNCELLE, M., Intersubjectivité et ontologie..., pp. 233-234.
}

77 Nédoncelle, M., Intersubjectivité et ontologie..., p. 326.

78 NÉdoncelle, M., Intersubjectivité et ontologie..., p. 331. 


\section{Si el amor es un 'trascendental' personal}

Una advertencia previa en este punto: si se hace coincidir el amor con una forma de amistad -como lleva a cabo alguno de los estudiosos de Nédoncelle- ${ }^{79}$ no se podrá sostener que el amor sea un 'trascendental' personal, porque, evidentemente, la amistad es una virtud (la más alta) de la voluntad, pero ésta es una 'potencia' y, como tal, no puede constituir el 'acto de ser' humano. En el fondo, aquí se pregunta si el amor en el ser humano es constitutivo o nativo, ${ }^{80}$ o si es, como la virtud de la amistad, una realidad adquirida.

a) En La reciprocidad de las conciencias, Nédoncelle mantuvo que el amor personal no está referido a las cualidades de la persona sino a la persona, ${ }^{81}$ pues "complacerse en las cualidades de una persona no es quererla, a menos que uno vea en estas cualidades la expresión y la manifestación queridas por la misma persona". ${ }^{82}$ En esta obra distingue también entre el amor de sentimiento y el amor de entrega. ${ }^{83}$ Agrega que "el amor no es la obra de uno o del otro de aquéllos a quienes une; ellos lo perciben o lo forman como el ser de su ser". ${ }^{44}$ Estas descripciones del amor parecen radicarlo en la intimidad personal. Afirma también que "el amor es lo único que puede suprimir la máscara", 85 lo cual indica que lo considera neurálgico, pues estima que puede

\footnotetext{
79 "La expresión máxima de la relación interpersonal es la amistad. La amistad es la díada más perfecta y el amor más grande que pueden tener las personas. La amistad es la que salva de la caducidad a los demás amores. Por esto se puede decir que es el más puro de los sentimientos humanos y el más profundo: conviene a todas las edades, es independiente de las condiciones sociales, tiende a penetrar completamente a aquellos que une, porque se dirige con una voluntad recíproca y generosa hacia el bien". VÁzquez BoraU, J. L., "La antropología de Maurice Nédonce1le", en Propuestas antropológicas del s. xx (I), J. F. Sellés (Ed.), Pamplona: Eunsa, 2ª ed., 2006, p. 367. Cf. asimismo de este autor: Del amor y la amistad. Platón-Nédoncelle, Barcelona: Horeb, 1997.
}

${ }^{80}$ En esta línea parecen ir las siguientes descripciones: "el amor es el verdadero sustrato ontológico de todos los procesos que se dan en el orden personal, siendo él, al mismo tiempo, lo más real y lo más personal. La realidad, el espíritu, lo concreto, se identifican con lo personal y con el amor que es todas esas dimensiones a la vez". FERNÁndez González, J., Antropología dialéctica. Estatuto metafísico de la persona según Maurice Nédoncelle, Tesis Doctoral, Madrid: Universidad Complutense de Madrid, 1982, Tomo II, 866. Más adelante añade: "el amor es el espíritu personal", p. 871; "la persona es amor", p. 872; "llegamos a la identificación de amor y persona", p. 876; "el amor es ser, antes de ser amor", p. 876; "el amor es la plenitud de la libertad interpersonal", p. 879; "el amor es esencialmente libertad ofrecida", p. 909; "El amor no es desligable de la persona", p. 926; "el amor pertenece a la esencia de la persona. No hay persona sin amor recíproco", p. 929; "el amor es la esencia espiritual de la comunión de los espíritus", p. 932. Cf. también: Canivez A., “La doctrine de l'amour chez Maurice Nédoncelle”, en du Congres sur le pensée de Maurice Nédoncelle, Paris: Tequi, 1981, pp. 113-122.

81 “Los dos extremos de la relación personal amorosa son sólo las personas y en cuanto personas, no en cuanto sujetos de cualidades o cuadro de valores". Fernández GonzÁLEz, J., Antropología dialéctica..., p. 893; “El amor alcanza y va dirigido, exclusivamente, al ser íntimo de la persona a la intimidad de la persona en cuanto ser". FERNÁNDEZ GonzÁlez, J., Antropología dialéctica..., p. 894.

82 Nédoncelle, M., La reciprocidad de las conciencias, p. 16.

${ }^{83}$ Cf. Nédoncelle, M., La reciprocidad de las conciencias, pp. 23-4.

${ }^{84}$ Nédoncelle, M., La reciprocidad de las conciencias, p. 26.

${ }^{85}$ Nédoncelle, M., La reciprocidad de las conciencias, p. 53. 
cambiar todo lo que es de orden manifestativo. Añade que el amor arrastra tras sí el conocimiento personal, ${ }^{86}$ pero éste se estima trascendental, ergo... En la vinculación amorosa este pensador habla del 'yo' y del 'tú', y aunque estas nociones no son del orden humano trascendental sino del manifestativo, tal como las describe parece aludir con ellas a la intimidad, que nunca es objeto sino acto: "amar al otro es buscar tornarlo amante, y si ya lo es, alegrarse de que lo sea". ${ }^{87}$ En este sentido se puede entender asimismo sus afirmaciones de que el amor es superior a la ética ${ }^{88}$ (siendo ésta de orden categorial), y de que el amor está por encima del pensar y del querer ${ }^{89}$ (que son, asimismo, predicamentales), porque radican en facultades humanas, las cuales, como 'potencias' que son, no se incluyen en el 'acto de ser', sino más bien en la 'esencia' humana.

b) Su obra Vers une philosophie de l'amour et de la personne está enteramente centrada en el tema del amor. "Una de las ideas directrices de este estudio -escribe Nédoncelle en el Prólogo- es que el amor procede de las personas y se dirige a ellas". ${ }^{90} \mathrm{Con}$ todo, lo describe como una 'voluntad de promoción'.${ }^{91}$ Como en este trabajo el autor sigue a Scheler y este fenomenólogo distinguía entre el amor sensible, el psíquico y el personal, ${ }^{92}$ cabría pensar que el filósofo de París entiende el amor personal -de modo similar al pensador de Munich- como trascendental. Sin embargo, Scheler no implicaba a la voluntad en el amor personal, por lo que hay que sostener que la coincidencia temática entre ambos no es completa en este punto, al menos porque no es explícito que el parisino considere al amor como un trascendental personal.

86 “El paro del amor es también el paro del saber". Nédoncelle, M., La reciprocidad de las conciencias, pp. 62-63. “La caridad exige una claridad recíproca", p. 183. "La caridad implica la verdad. Si pudiese realizarse completamente, realizaría la verdad, pues es el valor que engloba a los demás valores. Pero ella implica también la revelación de la verdad de una manera progresiva. Hace falta que el otro sea capaz de llevarla antes de que se la exprese, y esto es una condición dramática", p. 184. "No solamente el amor verdadero exige un perfecto conocimiento, sino que es la forma suprema de realidad", p. 241. "La connaissance réelle est un extrait de l'amour: elle veut à sa manière l'autre en tant qu'autre" p. 84. "Ĺamour réel contient la connaissance et la développe en son sein comme une germe indispensable à sa propre essence", p. 85.

${ }^{87}$ NéDoncelle, M., La reciprocidad de las conciencias, p. 85.

88 “Todo amor desborda la moralidad porque quiere también la reciprocidad". NÉDonCELLE, M., La reciprocidad de las conciencias, pp. 248. Sobre este punto Fernández González escribe que "el amor tiene que ser personal para sea 'moral' y pueda así fundar una ética personalista". Fernández González, J., Antropología dialéctica..., p. 922.

89 "La plenitud (del acto libre) está en el acto de amar, como síntesis de los actos de querer y pensar. No es comprendido por ellos, sino que los rebasa y comprende". Nédoncelle, M., La reciprocidad de las conciencias, p. 303.

${ }^{90}$ NéDoncelle, M., Vers une philosophie de l'amour et de la personne, p. 7.

91 “L'amour est une volonté de promotion”. NéDonCelLe, M., La reciprocidad de las conciencias, p.15. Vouloir qu'autrui soit aimant, c'est vouloir qu'il aime en moi ce par quoi je peux et je veux l'aimer; c'est vouloir qu'il m'aime", p. 32. "Par ce vouloir, les sujets s'identifient, et ils ne le font que dans la mesure où ils se diversifient", p. 44. "La volonté d'aimer implique celle d'être aimé", p. 62. "Toute relation du moi au toi est l'amour, c'est-à-dire la volonté de promotion mutuelle", p. 243. Esta reiterativa tesis suya es opuesta a la de Sartre. Cf. NéDoncelle, M., La reciprocidad de las conciencias, p. 243.

${ }^{92} \mathrm{Cf}$. al respecto mi trabajo: "Intuición y perplejidad en la antropología de Scheler", en Cuadernos de Anuario filosófico, Pamplona: Servicio de Publicaciones de la Universidad de Navarra, Serie Universitaria, núm. 216, 2009, pp. $42-48$. 
También admite que la reciprocidad es requerida en el amor ${ }^{93}$ y que éste requiere, además, obras, es decir, dones, los cuales tienen como fin consolidar la reciprocidad amorosa. ${ }^{94} \mathrm{Si}$ bien vincula el amor a la voluntad, no obstante afirma que es lo más excelso de esta facultad. ${ }^{95}$ En algunos pasajes -más al estilo de Pascal- lo vincula al corazón. ${ }^{96} \mathrm{Si}$ en la precedente obra ha indicado que es el amor el que tira del conocer, ahora, no sólo mantiene esa tesis, ${ }^{97}$ sino que añade que es el norte de la libertad: "el amor no quiere la personalidad libre por su libertad; quiere la libertad porque ésta es la condición de una personalidad verdaderamente amante". 98

c) En Conscience et logos. Horizons et méthodes d'une philosophie personnaliste, Nédoncelle apunta al corazón como sede del amor: "lo que un hombre tiene en propio no pasa por el interior de su juicio sino dentro de su sentimiento. El corazón, en el sentido de Scheler aún más que de Pascal, es nuestro solo recurso en este dominio". ${ }^{99}$ Pero si es dudoso que el corazón equivaliese para Pascal a la voluntad, es claro que en Scheler no se puede aceptar tal equivalencia. ${ }^{100}$

93 "L'amour relie les consciences dans une communauté spirituelle". Vers une philosophie de l'amour et de la personne, p. 41. "La relation y prend la forme de un nous", p. 41. "Le nous de l'amour est une identité hétérogène du moi et du toi. C'est la communauté de deux sujets en tant que sujets", p. 43. "La véritable identité est hétérogène. C'est-àdire qu'elle suppose la diversité des consciences et leur caractère irremplaçable", p. 44. De este tema se ha hecho eco Milolo en su tesis doctoral: Shambuti Milolo, La personne dans la dynamique de la réciprocité. Une approche de la philosophie de l'amour chez Maurice Nédoncelleedeentras quene dialectique qui , Roma: Université Pontificale Salesiana, 2006. Fernández González escribe al respecto que "no es, por consiguiente, la generosidad la suprema dimensión del amor. La generosidad como entrega de una de las partes, de las personas, a la otra, tiene que dejar paso a la reciprocidad como forma más ontológica y moral del ejercicio del amor". Fernández González, J., Antropología dialéctica..., p. 928. Y añade: "la reciprocidad no le viene a la persona de alguna razón fuera de sí misma, por compromiso, por encuentro, por comunión, o por contrato o acuerdo personal", p. 929.

94 "L'oeuvre aura pour bu de consolider la réciprocité déjà acquise". Scheller, Vers une philosophie de l'amour..., p. 68. En virtud de los dones cabe distinguir, como lleva a cabo Fernández González, el amor de nivel personal del amor a nivel de naturaleza humana: "si a nivel de individuo y de naturaleza, la dirección egoísta del amor es más fuerte y más espontánea, no sucede así a nivel de persona-comunión. En la metafísica de la persona, del ser-en-sien-mí-para-otro, el amor como don y élan personal, el amor estructura oblativa y deferente consagra la intuición del otro, es más fuerte que el egoísmo y más radicalizado en la esencia de la persona, aunque no en su psiquismo. El amor es la dimensión más relevante en una metafísica de la persona". Fernández González, J., Antropología dialéctica..., p. 915.

95 “Il n'est volonté parfaite qu'en étant parfait amour". Fernández González, J., Antropología dialéctica..., p. 86.

96 "C'est donc bien à l'intime subsistance de l'intellect e du vouloir dans l'amour que nous devons conclure". FerNÁNDEZ González, J., Antropología dialéctica..., p. 88; “la présence simultanée de l'amour et de la connaissance ou du vouloir est nécessaire". Fernández González, J., Antropología dialéctica..., p. 89.

97 “L'amour veut l'influence d'une conscience sur une autre”. Fernández González, J., Antropología dialéctica..., p. 95. “Les amants n'y trompent pas: ils savent très bien quand ils sont liés par un don mutuel”. Fernández GonZÁLEZ, J., Antropología dialéctica..., p. 96.

98 “L'amour ne veut pas la personnalité libre pour sa liberté; il veut la liberté parce qu'elle est la condition d'une personnalité vraiment aimante". Fernández GonzÁlez, J., Antropología dialéctica..., p. 93.

${ }^{99}$ NÉDoncelle, M., Conscience et logos..., p. 32.

${ }^{100}$ Cf. sobre la equivalencia de los términos 'corazón' y 'persona' en Scheler, mi aludido trabajo: SelLés, Intuición y perplejidad en la antropología de Scheler, p. 214. 
d) En Personne humaine et nature. Étude logique et métaphysique, Nédoncelle sigue vinculando el amor a la voluntad, y hace de él la clave de toda relación interpersonal: "la esencia de toda relación de mí y de ti es el amor, es decir, la voluntad de mutua promoción". ${ }^{101} \mathrm{Y}$, al igual que en las obras anteriores, subordina el conocer al amar: "conocer a otro es quererlo en su originalidad y en su libre espontaneidad". ${ }^{102}$ Pero no es claro en esta obra que el autor encuadre al amor en el ámbito trascendental.

e) En Explorations personnalistes Nédoncelle escribe que "la reciprocidad de las conciencias no se realiza plenamente más que en el amor, y esto es esencialmente intersubjetivo". ${ }^{103}$ En este libro sigue vinculando el amor a la voluntad: "la voluntad amante implica una voluntad de engendrar el ser amado". ${ }^{104}$ Añade que el amor ejerce una modificación del amante sobre el amado y viceversa. ${ }^{105}$ Como en obras precedentes, indica que el amor promueve el conocimiento intuitivo de las personas. ${ }^{106}$ También admite la convergencia entre el amor y la libertad personales. ${ }^{107} \mathrm{Y}$ como en las obras precedentes, tampoco en ésta es clara la inclusión del amor en el plano trascendental.

f) Por último, en Intersubjectivité et ontologie. Le défi personnaliste, Nédoncelle trata de la intersubjetividad a nivel manifestativo, no en el plano coexistencial o trascendental. Indica que algunos -como Sartre- basan esa vinculación social en el instinto, mientras que otros -como él mismo- la fundamentan en el amor, ${ }^{108}$ pues sólo éste puede unificar las relaciones personales. ${ }^{109} \mathrm{~A}$ esto, en el Apéndice de esta obra, titulado "Remarque finale: les deux formes de la relation", que es de 1974, añade que "en la comunicación hay en efecto un amor que promueve las unicidades personales... Es la más compleja y alta de las relaciones, la que nos establece en otro, en Dios sin rebajarnos al rango de atributos o de adjetivos los unos a los otros". ${ }^{110}$ Pero,

101 "L'essence de toute relation du moi au toi est l'amour, c'est-à-dire la volonté de promotion mutuelle". NÉDoNCELLE, M., Conscience et logos..., p. 29.

102 "Connaître autrui, c'est le vouloir dans son originalité concrète et dans sa libre spontanéité". NÉDonCELLE, M., Conscience et logos..., p. 29.

103 “La réciprocité des consciences ne se réalise pleinement que dans l'amour et celui-ci est essentiellement intersubjectif". NéDONCELLE, M., Explorations personnalistes, p. 85.

104 “Le volonté aimante implique une volonté d'engendrer l'être aimé". NÉDONCELLE, M., Explorations personnalistes, p. 85. Cf. asimismo: NédonCelle, M., Explorations personnalistes, p. 87.

${ }^{105}$ Cf. NédonCelle, M., Explorations personnalistes, p. 85.

106 “L'intériorité de l'amour surmonte le retard de la perception et permet parfois l'intuition difficile de l'absolu personnel dans son absolu éternel". NÉDONCELLE, M., Explorations personnalistes, p. 88.

107 “Il y a convergence entre l'amour, la liberté et l'éternité des personnes". NÉDonCELLE, M., Explorations personnalistes, p. 88.

108 Cf. NÉDoncelLe, M., Intersubjectivité et ontologie..., p. 15.

109 "Seul l'amour pet unifier ces relations". NÉDONCELLE, M., Intersubjectivité et ontologie..., p. 17.

${ }^{110}$ NÉDONCELLE, M., Intersubjectivité et ontologie..., p. 376. 
como se ve, tampoco aquí es patente que considere al amor como un trascendental personal; mientras que lo deleitable, afirmaba, solo en cierto modo resulta valioso en sí mismo. Por el contrario, desde una perspectiva más radical, también es relativo a quien desea disfrutar de él. El único bien radical y plenamente absoluto es el digno... también con las distinciones a que más tarde aludiré.

\section{Conclusiones}

Tras el estudio de la libertad, el conocimiento y el amor personales en las seis obras antropológicas más representativas de Nédoncelle respecto de estos temas, se pueden sacar las siguientes escuetas inferencias:

1. Aunque en algunos textos Nédoncelle vincula la libertad a la voluntad, en otros afirma que "mi libertad es mi persona". De manera que, al menos en esta segunda serie de pasajes, y asimismo en la explicación que lleva a cabo de la libertad nativa y de destino, se puede sostener que la enmarca en el ámbito trascendental.

2. Nédoncelle, que niega la posibilidad del conocimiento de la interioridad personal a través de los sentidos y de la razón en cualquiera de sus actos (el conocer objetivo, el concepto, el juicio, la analogía, los símbolos, etc.), tantea diversos métodos noéticos de acceso a dicha intimidad: la conciencia, la reflexión, la intuición intelectual, la $f e$, el sentimiento. Aunque vincula tal saber al orden del ser, no es explícito que tal saber sea de orden trascendental, pues indica que no coincide enteramente con el ser.

3. Nédoncelle vincula el amor a la voluntad; y a pesar de la relevancia que da al tema del amor, en especial de cara a las relaciones interpersonales, no es explícito que lo considere como un trascendental personal.

Estas tres intuiciones básicas sobre la libertad, el conocer y el amar, que Nédoncelle advirtió seguramente en algunas obras de Scheler, juntamente con la apertura o relación que denota el ser personal, ${ }^{111}$ abren la puerta a desarrollar y perfilar mejor una 'antropología trascendental', 112 es decir, a notar que la libertad coexistencial, el conocer y el amor personales son dimensiones constitutivas del acto de ser personal.

111 “La comunicación que la persona ofrece de sí misma es doble: en primer lugar, el resplandor o la transparencia que le permite conocer y amar sin saberlo; más brevemente dicho, su venida y presentación en la existencia. En segundo lugar, el don voluntario que ella hace de su propia existencia y que lleva a acoger en ella el acto del otro". NéDoncelle, M., La réciprocité des conscientes, Paris: Aubier-Montaigne, 1942, p. 312.

$112 \mathrm{Tal}$ antropología, sin previo conocimiento de la propia de Nédoncelle, se encuentra formulada y depurada en: Polo, L., Antropología trascendental (I). La persona humana, Pamplona: EunsA, 1999. 